\title{
Endophytic fungal isolate mediated biosynthesis of silver nanoparticles and their free radical scavenging activity and anti microbial studies
}

\author{
Vasudeva Reddy Netala ${ }^{1}$. Venkata Subbaiah Kotakadi $^{2} \cdot$ Pushpalatha Bobbu $^{1}$ • \\ Susmila Aparna Gaddam ${ }^{3}$ V Vijaya Tartte ${ }^{4}$
}

Received: 5 January 2016/Accepted: 24 May 2016/Published online: 10 June 2016

(c) The Author(s) 2016. This article is published with open access at Springerlink.com

\begin{abstract}
The present study reports that the biosynthesis of AgNPs using an endophytic fungus isolated from the ethnomedicinal plant Centella asiatica. The endophytic fungus was identified as Aspergillus versicolor ENT7 based on $18 \mathrm{~S}$ rRNA gene sequencing (NCBI Accession number KF493864). The AgNPs synthesized were characterized by UV-visible spectroscopy, Fourier transform infra-red spectroscopy (FTIR), transmission electron microscopy (TEM), X-ray diffraction (XRD), particle size analyzer, and zeta potential measurements. The UV-Vis absorption spectra showed the peak at $429 \mathrm{~nm}$ which confirmed the synthesis of AgNPs. TEM analysis revealed that the AgNPs were spherical in shape with $3-40 \mathrm{~nm}$ in size; similar results were also obtained by Horiba particle size analyzer with 5-40 nm in size. The synthesized AgNPs were highly stable due to their high negative zeta potential value of $-38.2 \mathrm{mV}$. XRD studies showed (111), (200), (220), (311), and (222) planes of the face-centered cubic (FCC) lattice, indicating the crystalline nature of the AgNPs. Selected area electron diffraction (SAED) pattern of the AgNPs showed five circular fringes which were in accordance with
\end{abstract}

Venkata Subbaiah Kotakadi

kotakadi72@gmail.com

$\bowtie$ Vijaya Tartte

tvijayasvu@yahoo.com

1 Department of Biotechnology, Sri Venkateswara University, Tirupati, AP, India

2 DST-PURSE Centre, Sri Venkateswara University, Tirupati 517502, AP, India

3 Department of Virology, Sri Venkateswara University, Tirupati, AP, India

4 Department of Botany, Sri Venkateswara University, Tirupati 517502, AP, India
XRD data and confirmed the formation of high crystalline nature of AgNPs. FTIR measurements indicated the peaks at $3273,2925,1629,1320$, and $1020 \mathrm{~cm}^{-1}$ corresponding to different functional groups possibly involved in the synthesis and stabilization of AgNPs. The synthesized AgNPs exhibited effective free radical scavenging activity with the IC50 value of $60.64 \mu \mathrm{g} / \mathrm{ml}$. The synthesized AgNPs were found to be highly toxic against both grampositive and gram-negative bacteria and also showed a very good antifungal activity.

Keywords 18S rRNA gene - Aspergillus versicolor . AgNPs - Zeta potential - Free radical scavenging activity · Antimicrobial activity

\section{Introduction}

Over the last decade majority of the researchers shifted their focus to nanometer-sized particles, particularly metal nanoparticles because of their controllable size and shape, ease of synthesis, and strong optical properties. The unique size- and shape-dependent properties of metal nanoparticles have significantly impacted all spheres of human life and making nanobiotechnology a promising field (Ravindran et al. 2013). Nanobiotechnology find innumerable applications due to strong optical absorbance and surface bioconjugation with molecular biomarkers related to surface plasmon resonance effect (Kreibig and Vollmer 1995). Noble metal nanoparticles particularly silver nanoparticles (AgNPs) have gained a significant interest in the recent years, owing to their remarkable optical, optoelectronic, magnetic, catalytic, and thermal properties and thus find applications as optical receptors (Karimzadeh and Mansour 2010), sensors (Cobley et al. 2009), catalysts in chemical 
reactions (Edison and Sethuraman 2012), signal enhancers in SERS-based enzyme Immunoassay (Chen et al. 2009), DNA detection and delivery, bio-analyzers (Ravindran et al. 2013), Cytotoxic (Asad et al. 2013), free radical scavenging (Ramamurthy et al. 2013), and antimicrobial agents (Netala et al. 2014, 2015a; Kotakadi et al. 2013).

Several physical and chemical approaches have been reported for the biosynthesis of silver nanoparticles, including thermal decomposition (Plante et al. 2010), laser ablations (Simakin et al. 2004), gamma radiation assisted (Chol et al. 2005), polyol assisted, and chemical reduction methods (Frattine et al. 2005). Irradiation and other physical methods are not environmental friendly and also possess health risks. Hazardous chemicals, such as hydroxylamine, poly- $N$-vinyl pyrrolidine, polyvinyl alcohol, and sodium borohydride, are involved in the chemical approaches. The application of chemical approaches is not eco-friendly and possesses biological risks, and the toxic chemicals on the surface limit the applications of AgNPs in pharmaceutical and biomedical fields. Hence, the biological methods for the synthesis of AgNPs have gained a significant interest in the field of nanotechnology. Supramolecular complexes of biomolecules and their topographic and electrostatic properties are being widely employed in biomedical and pharmaceutical fields. Peptides to proteins, sugars to polysaccharides, isoprenes to terpenoids, polyphenols, glycosides, plant and microbial derived compounds, viral particles, etc., are being constantly explored for the biosynthesis of metal nanoparticles and novel carriers. Biological approaches are simple, rapid, and cost effective and involve the synthesis of non-toxic, clean, and biocompatible AgNPs. Fungal mediated synthesis of AgNPs has recently emerged as a new avenue in the field of nanobiotechnology. Compared to bacteria, fungi are more advantageous, because they grow at fast rates and very easy to culture, and maintain in the laboratory. Fungi being eukaryotes produce high amounts of proteins and other biomolecules. These proteins and biomolecules will often associate with nanoparticles that must be preventing the agglomeration and stabilize nanoparticles. Various fungi have been reported to synthesize AgNPs, including Alternaria alternate (Monali et al. 2009), Amylomyces rouxii (Javed et al. 2010), Aspergillus niger (Netala et al. 2015b), Coriolus versicolor (Rashmi and Varma 2009), Fusarium solani (Rafie et al. 2012), Humicola sp (Asad et al. 2013), Neurospora crossa (Longoria et al. 2011), Schizophyllum radiatum (Metuku et al. 2014), and Trichoderma viride (Fayaz et al. 2009) have been reported.

Endophytic fungi are the intriguing group of fungal species which colonise living and healthy tissues of plants. Endophytic fungi produce natural bioactive compounds which are considered to be alternative sources for plant producing bioactive compounds (Strobel et al. 2002). Exploration of endophytic fungi for the biosynthesis of AgNPs is considered as their another important application in the pharmaceutical and biomedical field. In the present study, we report the biosynthesis of AgNPs using extracellular filtrate of endophytic fungus Aspergillus versicolor ENT7 strain isolated from the healthy leaf tissues of Centella asiatica, an important medicinal plant which harbors many endophytic fungi. The AgNPs were characterized using different measurements which include UVVis, FTIR, XRD, TEM, particle size analyzer, and zeta potential measurements. Biomedical importance of the AgNPs was evaluated by checking for free radical scavenging and antimicrobial activities.

\section{Materials and methods}

\section{Isolation of endophytic fungi}

Mature leaves of Centella asiatica were collected from plants grown in the Sri Venkateswara University campus, Tirupati, A.P. India. Leaves were washed under running tap water and then with teepol solution to ensure for dust free and clean. Then, leaves were washed thoroughly with sterile double distilled water (SDDW). Under aseptic conditions, leaves were surface sterilized with $10 \% \mathrm{H} 2 \mathrm{O} 2$ and then with $80 \%$ alcohol followed by thorough rinsing with SDDW for three to four times. Leaves were dried on sterile blotting paper and then cut into small segments. Leaf segments were placed on solidified potato dextrose agar (PDA) plates. PDA plates were incubated at $24 \pm 2{ }^{\circ} \mathrm{C}$ for 10 days. After 10 days, fungal mycelia were harvested and transferred onto fresh PDA plates. Pure fungal cultures were then identified by $18 \mathrm{~S}$ rRNA gene amplification and sequencing.

\section{Identification of endophytic fungi}

\section{DNA extraction}

For the extraction of genomic DNA, $50 \mathrm{mg}$ of fungal mycelia was frozen in liquid nitrogen and mechanically disrupted. The extraction of genomic DNA was carried out using Qiagen kit (USA) according to manufacturer's instructions.

\section{PCR amplification and sequencing of $18 S$ rRNA gene}

The ITS region (ITS 1-5.8S-ITS 2) of 18S rRNA gene was amplified using fungal domain specific primers ITS $1-5^{\prime}-\mathrm{T}$ CCGTAGGTGAACCTGCGG- $3^{\prime}$ (forward primer) and ITS4-5'-TCCTCCGCTTATTGATATGC-3' ${ }^{\prime}$ (reverse 
primer). Amplification was performed in a $50 \mathrm{ul}$ reaction mixture containing $50 \mathrm{ng}$ of template DNA, $200 \mu \mathrm{M}$ each dNTP, $1.5 \mathrm{mM} \mathrm{MgCl}_{2}, 20 \mathrm{pmol}$ of each primer, and $0.4 \mathrm{U}$ of Taq DNA polymerase in a CG palm cycler (Genetix biotech asia). The amplification cycles consisted of the initial denaturation at $94{ }^{\circ} \mathrm{C}$ for $5 \mathrm{~min}$ followed by 30 cycles of $94{ }^{\circ} \mathrm{C}$ for $45 \mathrm{~s}, 55^{\circ} \mathrm{C}$ for $1 \mathrm{~min}, 72{ }^{\circ} \mathrm{C}$ for $1 \mathrm{~min}$, and a final extension at $72{ }^{\circ} \mathrm{C}$ for $7 \mathrm{~min}$. The amplification was confirmed by running the amplified product in $1.2 \%$ w/v agarose gel electrophoresis with ethidium bromide staining and documented by gel documentation system (Major science, UVDI). The PCR products were purified using gel extraction kit. Sequencing was carried out at MWGAG Biotech, Bangalore, India. The sequence obtained was analyzed by BLASTn and NCBI and was identified by homology search for closely related sequence. Multiple sequence alignment was carried out using ClustalW2, and phylogenetic tree was constructed using the neighbor-joining (NJ) method.

\section{Biosynthesis of AgNPs}

The synthesis of silver nanoparticles was carried out according to the method described earlier (Jaidev and Narasimha 2010). The fungal isolate Aspergillus versicolor was cultured in $100 \mathrm{ml}$ of potato dextrose broth and was incubated at $26 \pm 2{ }^{\circ} \mathrm{C}$ in shaking incubator (LABLINE) at a speed of $100 \mathrm{rpm}$. After 7 days of growth, the fungal biomass was harvested and washed thoroughly with SDDW to prevent the contamination of medium components. $10 \mathrm{~g}$ of fungal biomass was taken in $250 \mathrm{ml}$ Erlenmeyer flask containing $100 \mathrm{ml}$ SDDW and incubated at $28 \pm 4^{\circ} \mathrm{C}$ for $72 \mathrm{~h}$ in shaking incubator at the speed of $100 \mathrm{rpm}$. After incubation, the aqueous solution was separated by filtration through Whatmann No.1 filter paper. This solution, namely, fungal filtrate, used for the synthesis of AgNPs. $100 \mathrm{ml}$ of $1 \mathrm{mM}$ of silver nitrate was then added to $100 \mathrm{ml}$ of fungal filtrate and incubated at $28 \pm 4{ }^{\circ} \mathrm{C}$ for $24 \mathrm{~h}$ in the dark condition.

\section{Characterization of AgNPs}

The synthesis of AgNPs was confirmed by the absorbance spectrum using UV-Vis spectrometer (Analytical Technologies Ltd, India). The spectrum was recorded with a resolution of $1 \mathrm{~nm}$ between 200 and $700 \mathrm{~nm}$. The synthesized AgNPs were purified by the centrifugation of solution of AgNPs at 15,000 rpm for 15 min thrice with continuous washing the pellet with sterile Milli $Q$ water. The final pellet that obtained was dried in hot air oven at $50{ }^{\circ} \mathrm{C}$ for $24 \mathrm{~h}$, and the pure powder obtained was used for FTIR, XRD, TEM, and other studies. FTIR analysis was carried out to study the functional groups possibly involved in the synthesis and stabilization of AgNPs. FTIR spectrum (Model ALPHA interferometer) was recorded in the range of $500-4000 \mathrm{~cm}^{-1}$ with the resolution of $2 \mathrm{~cm}^{-1}$. XRD patterns were obtained on an Ultima IV X-ray powder diffractometer (Rigaku, Tokyo, Japan) using $\mathrm{CuK} \alpha$ radiation $(\lambda=1.5406)$. The shape and size of the AgNPs were determined using TEM (FEI Tecnai F12, Philips Electron Optics, Holland) operated at $100 \mathrm{kV}$. Particle size and zeta potential experiments were carried out using a Horiba Nanopartica instrument.

\section{Free radical scavenging activity}

The free radical scavenging activity of the AgNPs was measured in vitro by $2,2^{\prime}$-diphenyl-1-picrylhydrazyl (DPPH) radical scavenging assay according to the method described earlier (Mittal et al. 2006). The stock solution was prepared by dissolving $4 \mathrm{mg}$ of DPPH in $100 \mathrm{ml}$ of methanol and stored at $20{ }^{\circ} \mathrm{C}$. $2 \mathrm{ml}$ of this solution was added to $1 \mathrm{ml}$ of methanol solution containing tested samples of different concentrations $(25,50,75$, and $100 \mu \mathrm{g} / \mathrm{ml}$ ). Ascorbic acid was used as standard. DPPH radical scavenging activity (RSA) was measured by the absorbance at $517 \mathrm{~nm}$

RSA $(\%)=[($ control absorbance - sample absorbance $) /$ (control absorbance) $] \times 100$.

\section{Antimicrobial activity}

Antimicrobial activity of the biosynthesized AgNPs was checked against both bacterial and fungal pathogens. Antibacterial activity of the AgNPs was checked against most pathogenic Staphylococcus aureus, Streptococcus pneumonia (gram positive), Pseudomonas aeruginosa, and Klebsiella pneumonia (gram negative) by employing the disc diffusion method using $24 \mathrm{~h}$ active cultures (Pulicherla et al. 2015). In the present study, bacterial cultures showed that the optical density (OD) value of 0.2 at $600 \mathrm{~nm}$ wavelength, according to McFarland calculation bacterial cell density, in this study is determined as $6 \times 10^{7}$ CFU/ml. $200 \mu \mathrm{l}$ of bacterial inoculum was spread on nutrient agar (NA) plates. Sterile paper disc dipped in $25 \mu \mathrm{l}$ (1 mg/1 ml) of AgNPs solution was placed on agar plates. Streptomycin was used as the standard antibiotic. NA plates were incubated at $37{ }^{\circ} \mathrm{C}$ for $24 \mathrm{~h}$ and observed for the zone of inhibition (ZOI).

Antifungal activity of the AgNPs was tested against the fungus Candida albicans and Candida nonalbicans using the disc diffusion method (Pulicherla et al. 2015). $200 \mu \mathrm{l}$ of fungal inoculum was spread on the potato dextrose agar (PDA) plates. Sterile paper disc impregnated with $25 \mu \mathrm{L}$ of AgNPs solution $(1 \mathrm{mg} / 1 \mathrm{ml})$ was placed on the PDA plate. 
Voriconazole was used as positive control. PDA plates were incubated at $25^{\circ} \mathrm{C}$ for $72 \mathrm{~h}$ to observe the inhibition zones.

\section{Results and discussion}

\section{Fungal identification using ITS rRNA gene sequencing and phylogenetic analysis}

The isolated endophytic fungus appeared as whitish brown in color (Fig. 1) and was characterized by the PCR amplification of $18 \mathrm{~S}$ rRNA gene using ITS primers. The amplified PCR product was around the size of 500bps. The Sanger's dideoxy nucleotide sequencing of amplified ITS region (ITS 1-5.8S-ITS 2) of $18 \mathrm{~s}$ rRNA gene resulted in 517 bp nucleotide sequence. The Blastn analyses, pairwise and multiple sequence alignment revealed 98-100\% identity with the sequences of $A$. versicolor strains and is designated as Aspergillus versicolor ENT 7 and has been deposited in NCBI GenBank (Accession Number KF493864). Multiple sequence alignment was carried out using ClustalW2 with default parameters. Phylogenetic tree was constructed by the neighbour-joining (NJ) method with nucleotide pairwise genetic distance corrections. Bootstrap test of phylogeny was carried out to check the reliability of tree topology as a percentage of 1000 replications. All branches with $<70 \%$ bootstrap support were collapsed (Fig. 2).

\section{Biosynthesis of AgNPs}

The synthesis of AgNPs by fungal filtrate of A. versicolor ENT 7 was investigated. After $24 \mathrm{~h}$ of incubation, light yellow color of the reaction mixture turned to dark brown color (Fig. 3). The color change indicated the reduction of

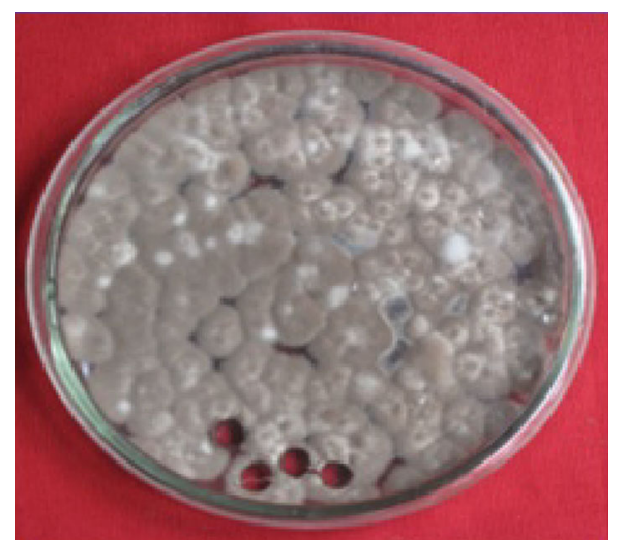

Fig. 1 Colony morphology of Aspergillus versicolor strain ENT 7 isolated from the leaves of Centella asiatica

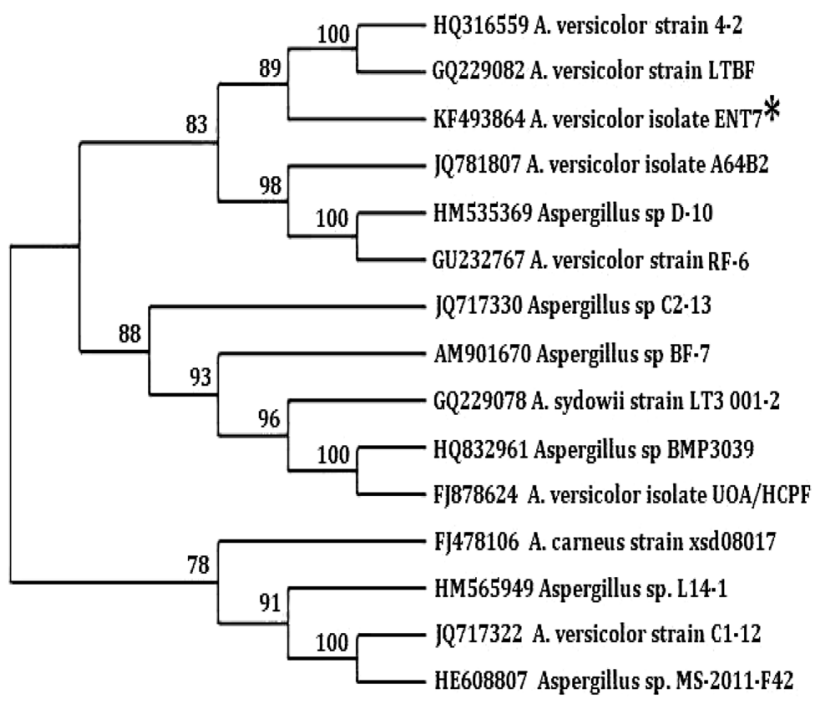

Fig. 2 Phylogenetic analysis of 18S rRNA gene of Aspergillus versicolor strain ENT 7 with other fungal isolates. [neighbour-joining (NJ) analyses of phylogenetic relationship between the A. versicolor isolate ENT7 (KF493864) and ITS-rDNA of related fungal strains retrieved from NCBI GenBank]

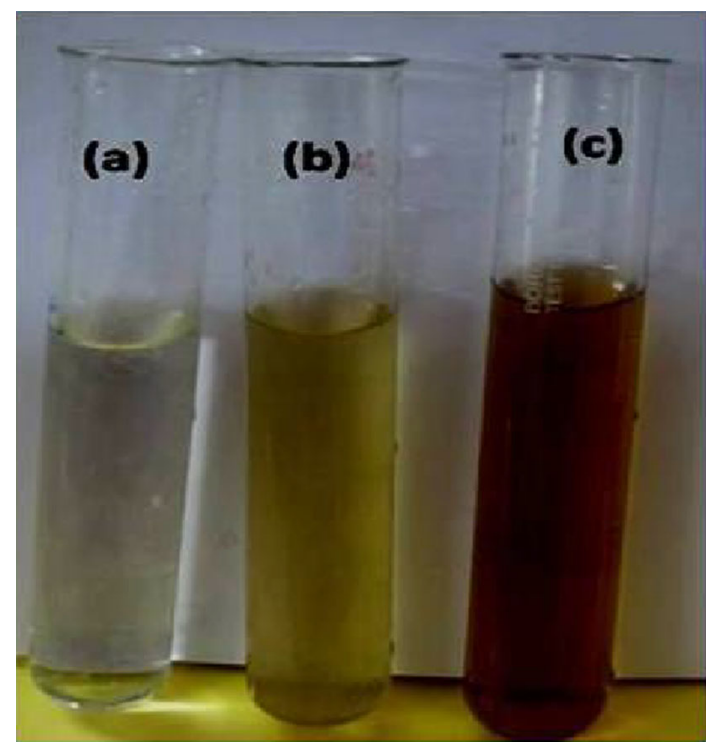

Fig. 3 a $1 \mathrm{mM}$ of $\mathrm{AgNO}_{3}$ solution. b Light yellow color of the reaction solution containing $1 \mathrm{mM} \mathrm{AgNO}_{3}$ and fungal filtrate. c Color change of the reaction solution from light yellow to dark brown indicating the formation of AgNPs

silver ions $\left(\mathrm{Ag}^{+}\right)$into $\mathrm{AgNPs}\left(\mathrm{Ag}^{0}\right)$. It is well known that AgNPs exhibit a dark brown color in aqueous solution due to surface plasmon resonance (SPR). The fascinating and bright colors of metal nanoparticles are related to the localized surface plasmon resonance (SPR). The applications of metal nanoparticles have broadened due to surface bioconjugation with molecular biomarkers strong optical absorbance related to SPR (Kreibig and Vollmer 1995; Kelly et al. 2002). 


\section{UV-visible spectra analysis}

The UV-visible spectra of synthesized AgNPs (Fig. 4) showed characteristic SPR peak at $429 \mathrm{~nm}$. SPR peak at $429 \mathrm{~nm}$ confirmed the synthesis of AgNPs and is also responsible for the spherical shape of AgNPs synthesized. The shape of the synthesized AgNPs is further confirmed by the TEM analysis. Different metabolites and proteins present in the extracellular fungal filtrate could be responsible for the synthesis and stabilization of AgNPs, which is further confirmed by the FTIR analysis.

\section{FTIR spectra analysis}

The FTIR analysis (Fig. 5) showed intensive peaks at 3273, $2925,1629,1320$, and $1020 \mathrm{~cm}^{-1}$. The peak at $3273 \mathrm{~cm}^{-1}$ corresponding to $\mathrm{N}-\mathrm{H}$ stretching of the secondary amide of the protein and the peak at $2925 \mathrm{~cm}^{-1}$ corresponding to C$\mathrm{H}$ stretching of methylene groups of the protein (Bozanic et al. 2010). Peak at $1629 \mathrm{~cm}^{-1}$ corresponding to $-\mathrm{CO}$

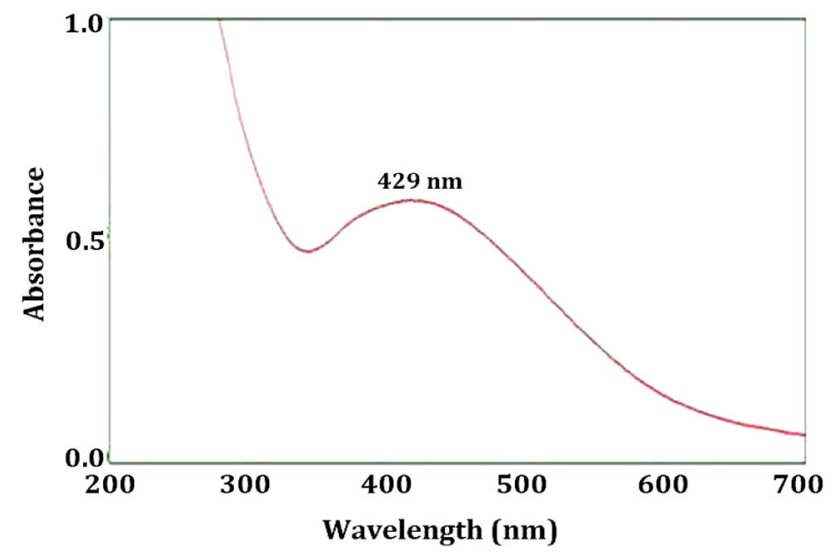

Fig. 4 UV-Vis analysis of the synthesized AgNPs showing SPR peak at $429 \mathrm{~nm}$

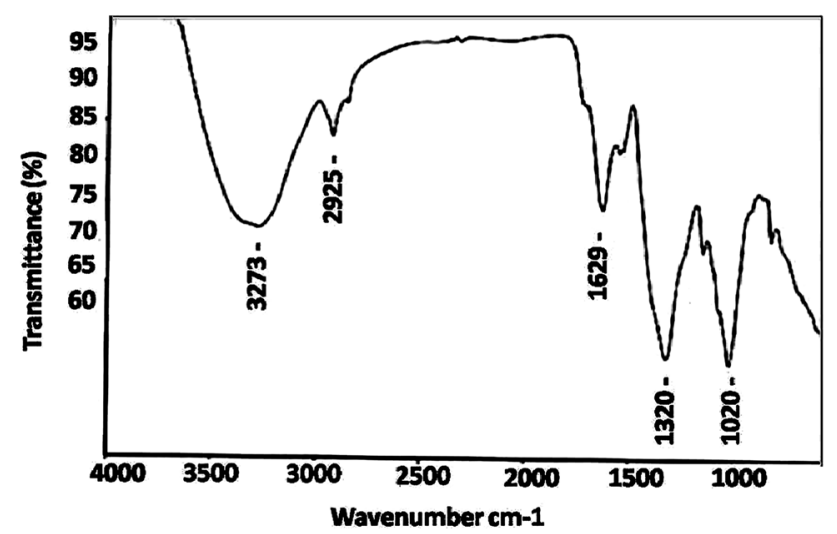

Fig. 5 FTIR analysis of biosynthesized AgNPs stretch of amide I band of proteins (Fayaz et al. 2009). The sharp peak at $1320 \mathrm{~cm}^{-1}$ can be assigned to $\mathrm{C}-\mathrm{N}$ stretching vibrations of aromatic amines (Rashmi and Varma 2009). The extracellular proteins present in the fungal filtrate could be responsible for the reduction of silver ions, $\mathrm{Ag}^{+}$ into nanosize silver particles, $\mathrm{Ag}^{0}$. These proteins have strong ability to bind silver nanoparticles, acts as capping agents and thus provided the stability to them. These results are consisted with the earlier reports for the fungal mediated synthesis of AgNPs (Jaidev and Narasimha 2010; Rafie et al. 2012: Asad et al. 2013). The peak at $1020 \mathrm{~cm}^{-1}$ can be assigned to $\mathrm{C}-\mathrm{OH}$ of the phenols, supporting the participation of polyphenols, such as flavanoids and triterpenoids, in the reduction of $\mathrm{Ag}^{+}$into $\mathrm{Ag}^{0}$ (Litvin et al. 2012; Litvin and Minaev 2013). FTIR analysis revealed that the polyphenols could act as bioreducing agents, while proteins could play a dual role as bioreducing and stabilizing agents.

\section{XRD analysis}

The XRD pattern of the biosynthesized AgNPs was shown in Fig. 6. XRD pattern revealed four diffraction peaks at $38.31^{\circ}, 44.58^{\circ}, 64.71^{\circ}, 77.71^{\circ}$, and $81.92^{\circ}$ could be indexed to (111), (200), (220), (311), and (222) planes, respectively. All the peaks corresponding to face-centered cubic (FCC) lattice phase of silver and were consisted with the standard JCPDS (File No 87-0719) data. Thus, XRD pattern obtained for the AgNPs that revealed the crystalline nature of AgNPs with FCC phase and consisted with many earlier reports of the AgNPs synthesized by fungal extracts (Rafie et al. 2012; Asad et al. 2013).

\section{TEM analysis}

TEM analysis was performed to determine the size and shape of the biosynthesized AgNPs. TEM micrographs were represented at different resolutions (Fig. 7a, c). TEM micrographs obtained shows that AgNPs were roughly

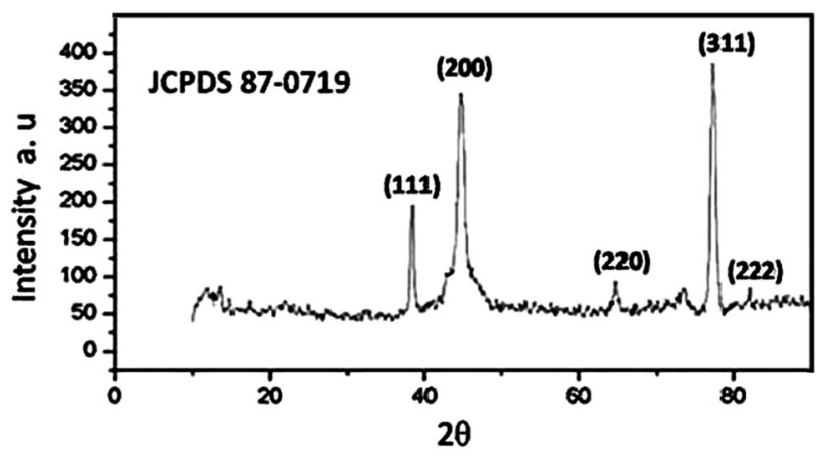

Fig. 6 XRD pattern of biosynthesized AgNPs 

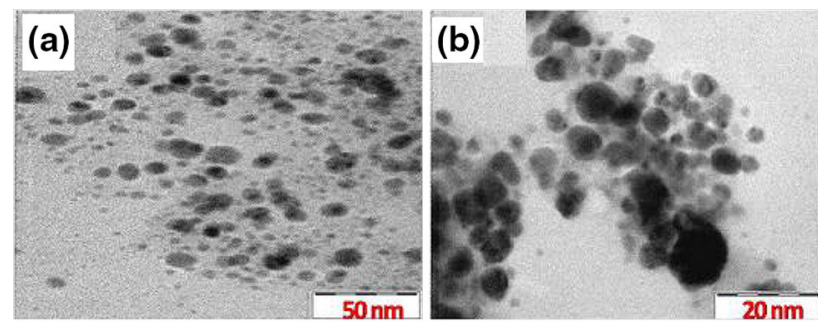

(c)
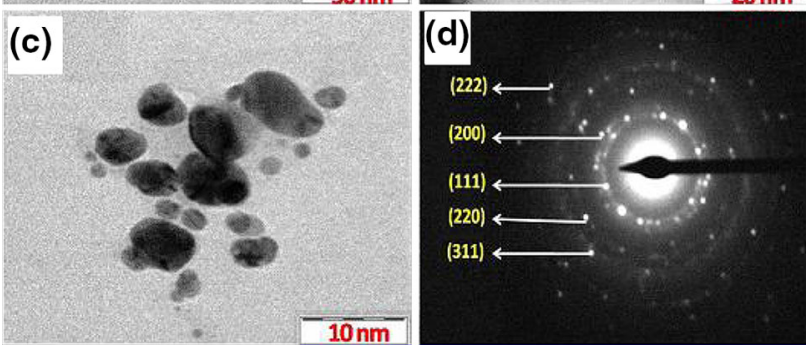

Fig. 7 TEM images of AgNPs at a $50 \mathrm{~nm} \mathrm{~b} 20 \mathrm{~nm}$ and c $10 \mathrm{~nm}$ and corresponding SAED pattern $\mathbf{d}$ showed five diffraction rings

spherical in shape and well dispersed with the sizes ranging from 3 to $40 \mathrm{~nm}$. From the TEM micrographs, it is evident that the size and shape of the AgNPs were fairly uniform. Selected area electron diffraction (SAED) pattern of the AgNPs (Fig. 7d) depicted circular fringes corresponding to (111), (200), (220), (311), and (222) planes which were in accordance with XRD data and further confirmed the formation of high crystalline AgNPs. The results of the TEM studies are in line with earlier reports (Metuku et al. 2014).

\section{Particle size determination}

The particle size of the biosynthesized AgNPs was detected by intensity and laser diffraction. After the analysis of AgNPs, it has been revealed that the particles synthesized with extracellular fungal filtrate are in the range of $5-40 \mathrm{~nm}$ in size. The average size (hydrodynamic radius) of AgNPs was found to be $15.5 \mathrm{~nm}$ (Fig. 8). The results are similar to that of TEM results indicating that the size of the AgNPs is uniform in nature. The biosynthesized AgNPs are poly-dispersed in nature. The stability was further confirmed by zeta potential of the particles.

\section{Zeta potential of AgNPs}

The electrostatic repulsive force between the nanoparticles depends on the charge which is present on the surface of the nanoparticles. In the present study, the very high negative value of zeta potential confirms the repulsion among the particles and thereby increases the stability of the formulation, and prevents the nanoparticles from agglomeration in the medium, leading to the long-term stability. The zeta potential of the AgNPs of fungal filtrate was found to be $-38.2 \mathrm{mV}$ (Fig. 9). Based on the above results, it is concluded that the AgNPs synthesized with A. versicolor fungal filtrate were very stable.

\section{Free radical scavenging activity}

Free radical scavenging activity of the AgNPs was checked by DPPH radical scavenging assay. This method is dependent on the reduction of DPPH radical to the nonradical form DPPH-H in the presence of a hydrogen-donating antioxidant. The radical scavenging activity and $\mathrm{IC}_{\mathbf{5 0}}$ values of all the tested samples were represented in Table 1 . The radical scavenging activity was increased with the increasing concentrations of tested samples. The radical scavenging activity for the AgNPs at $100 \mu \mathrm{g} / \mathrm{ml}$ was determined as $60.04 \%$ which is close to $68.52 \%$ obtained for the standard ascorbic acid at the same concentration. However, the radical scavenging activity for the fungal filtrate was found to be $41.19 \%$. Thus, the biosynthesized AgNPs were proved to be very effective scavengers. The radical scavenging activity was also expressed as $\mathrm{IC}_{\mathbf{5 0}}$ (inhibitory concentration of the test sample to scavenge $50 \%$ radicals). $\mathrm{IC}_{\mathbf{5 0}}$ value for the AgNPs is found to be $60.64 \mu \mathrm{g} / \mathrm{ml}$, while same for the fungal filtrate was found to be $144.84 \mu \mathrm{g} / \mathrm{mL}$. The lower $\mathrm{IC}_{50}$ value means high radical scavenging activity of the sample. Thus, the biosynthesized AgNPs found to be very good antioxidants. Free radical scavenging activity of the AgNPs is mainly due to hydrogen-donating molecules, such as polyphenols, flavonoids, terpenoids, proteins, and other biomolecules, that are present in the colloidal solution of AgNPs or the secondary metabolites, proteins, and other biomolecules that are involved in the synthesis and stabilization of AgNPs. Silver nitrate could not show any free radical scavenging activity in our study. Silver nitrate does not have any hydrogen to participate in the scavenging of free radical DPPH. There were no reports also for the free radical scavenging activity of silver nitrate. Free radical scavenging activity studies of AgNPs biosynthesized in this study are in line with many earlier reports (Ramamurthy et al. 2013). Antioxidants are the compounds which protect cells against the cellular damaging effects of reactive oxygen species (ROS), such as hydroxyl radicals, peroxy radicals, peroxylnitrile, super oxide, and singlet oxygen. The lower concentration of antioxidants or the imbalance between antioxidants and ROS results in oxidative stress which could be linked to inflammation, neurodegenerative disorders, atherosclerosis, aging, and cancer (Ramamurthy et al. 2013). Based on our results, it is revealed that the AgNPs can be used as ingredients in the antioxidant formulations in pharmaceutical or biomedical field. The excellent radical scavenging activity of the AgNPs allowed them to be employed in the numerous applications, such as food preparation ingredients, food 
Fig. 8 Particle size analysis of biosynthesized AgNPs
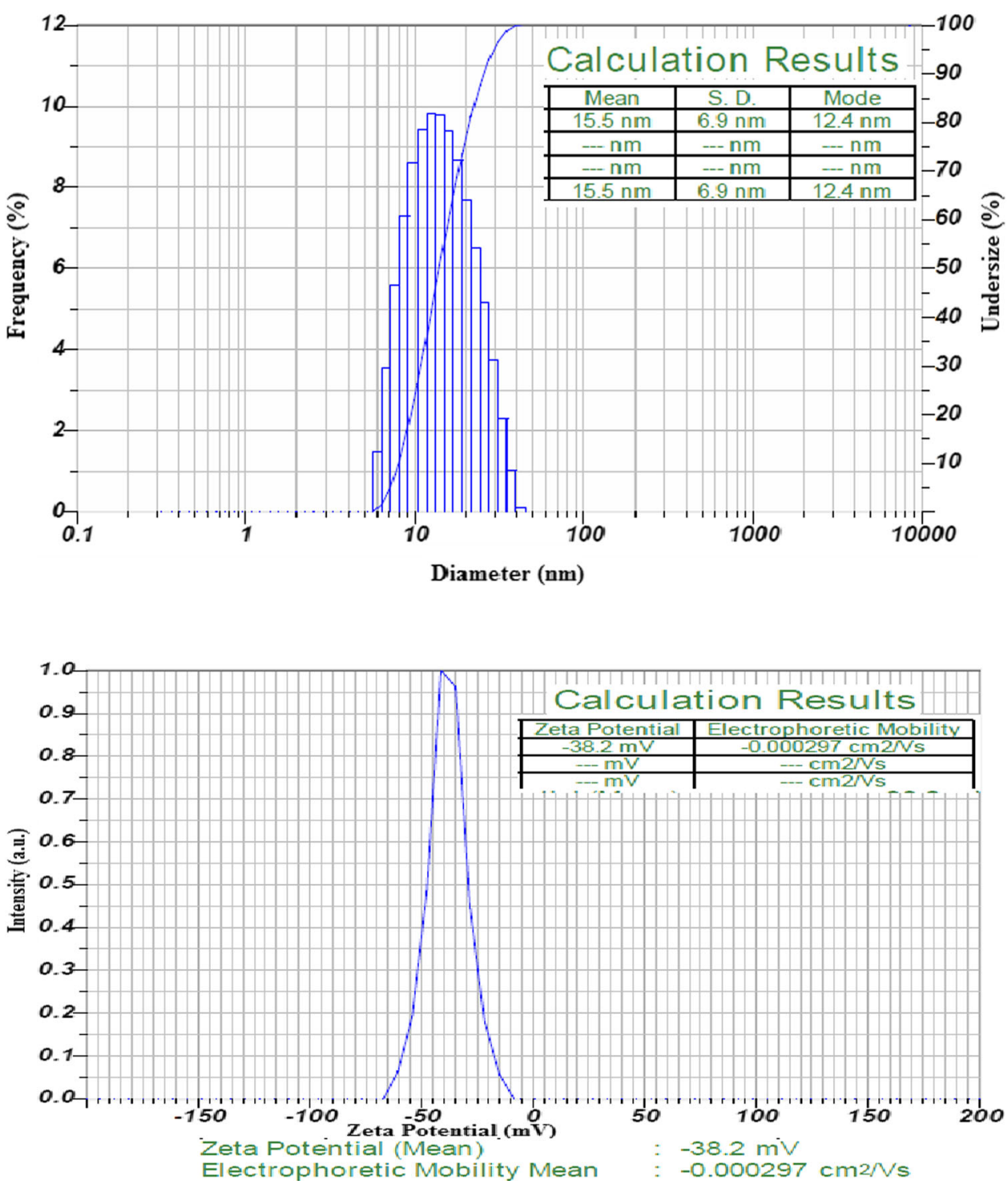

Fig. 9 Zeta potential analysis of biosynthesized AgNPs

Table 1 DPPH radical scavenging activities of AgNPs compared with fungal filtrate and standard ascorbic acid

\begin{tabular}{lllrr}
\hline Tested sample & \multicolumn{4}{l}{ Radical scavenging activity $\pm \mathrm{SD}(\%)$} \\
\cline { 2 - 5 } & $25(\mu \mathrm{g} / \mathrm{mL})$ & $50(\mu \mathrm{g} / \mathrm{mL})$ & $75(\mu \mathrm{g} / \mathrm{mL})$ & $100(\mu \mathrm{g} / \mathrm{mL})$ \\
\hline Ascorbic acid & $47.08 \pm 0.26$ & $55.62 \pm 0.25$ & $62.51 \pm 0.35$ & $68.52 \pm 0.67$ \\
Fungal filtrate & $27.05 \pm 0.42$ & $31.48 \pm 0.34$ & $37.05 \pm 0.51$ & $41.19 \pm 0.26$ \\
AgNPs & $39.28 \pm 0.25$ & $47.53 \pm 0.19$ & $55.39 \pm 0.44$ & $60.04 \pm 0.25$ \\
\hline
\end{tabular}

storage additives, vegetable oil preparations, and various syrup formulations.

\section{Antimicrobial activity}

After incubation for $24 \mathrm{~h}$, growth inhibition (ZOI) was observed around discs impregnated with AgNPs and streptomycin. Figure 10 shows the strong antibacterial activity of AgNPs against Staphylococcus aureus, Streptococcus pneumonia (gram negative), Pseudomonas aeruginosa, and Klebsiella pneumonia (gram negative); maximum ZOI was observed against Pseudomonas aeruginosa $(18.5 \mathrm{~mm})$ followed by Klebsiella pneumonia (13.6 mm), Staphylococcus aureus (12.1 mm), and Streptococcus pneumonia $(11.8 \mathrm{~mm})$. The results revealed that AgNPs showed strong inhibition against gram-negative 
Fig. 10 Antibacterial activity of the AgNPs against $S$. aureus (a), S. pneumonia (b), $P$. aeruginosa and $\mathbf{d} K$. Pneumonia. No inhibition zones formed by fungal filtrate (i) Very well inhibition zones were observed by Streptomycin (ii) and by AgNPs (iii)

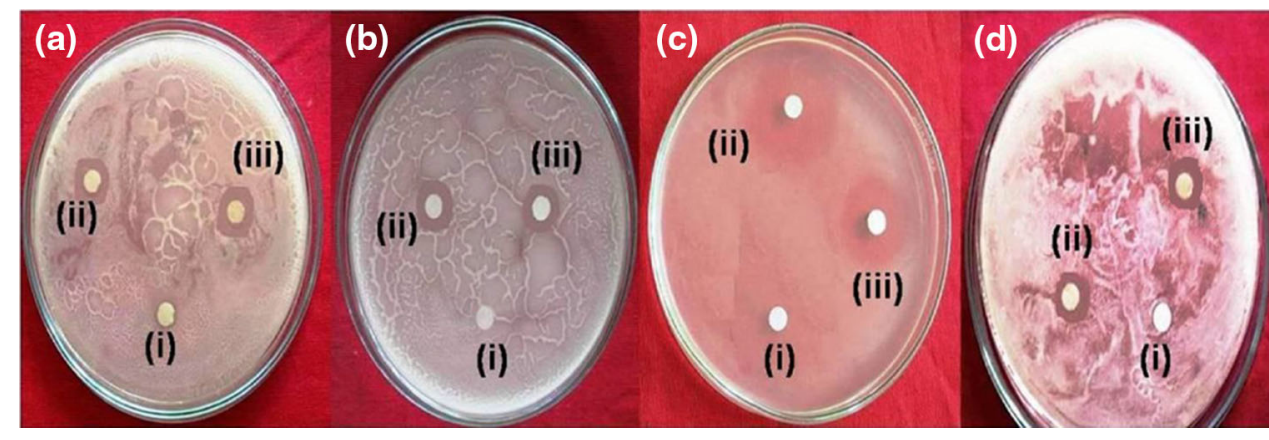

bacteria compared to gram-positive bacteria. Not only antibacterial activity, AgNPs also showed a very good antifungal activity against most pathogenic Candida albicans and Candida nonalbicans and formed the ZOI values of 12.2 and $13.6 \mathrm{~mm}$, respectively. The results revealed that the growth inhibitory activity of AgNPs against fungal pathogens can be increased further by increasing the concentration of AgNPs as these are clean, non-toxic, and biocompatible, but the drugs will be toxic and non-biocompatible at higher concentrations. The results of the antifungal activity were consisted with many earlier reports (Kim et al. 2009; Rafie et al. 2012). The antimicrobial activity of AgNPs is probably the sum of distinct mechanisms. AgNPs react with thiol (-SH) groups of vital enzymes/proteins and inactivates them, in turn stop the progress of cellular metabolisms which leads to cell death (Morones et al. 2005; Netala et al. 2015b). AgNPs form strong interaction with proteins of the proton pump and phospholipid portion of the bacterial membrane resulted in the dissipation of membrane proton gradient causing disruption of bacterial membranes (Kotakadi et al. 2014). AgNPs form strong interaction with respiratory chain enzymes which causes uncoupling of electron transport chain (ETC) and oxidative phosphorylation (OP) as a result cell death occurs (Morones et al. 2005; Feng et al. 2000). Proteomic analysis revealed that the exposure of bacteria to nanosilver resulted in alterations in the expression of envelope and HSPs, as a result AgNPs can penetrate and can disrupt the membranes and leads to leakage of protons, potassium ions and reducing sugars and eventually leads to death (Lok et al. 2006). The excellent antimicrobial activity of the AgNPs allows them to be used in ingredients of topical ointments to prevent bacterial and fungal infections against open and burn wounds, AgNPs embedded water purifier equipments, preparation of injection molded plastic products, and employed in coating-based applications, including air ducts and counter tops. The important advantages of AgNPs-based antimicrobial agents are their biocompatibility, health and environmental safety, and their excellent stability.

\section{Conclusion}

An endophytic fungal isolate, ENT7 was isolated from the leaves of Centella asiatica and was identified as Aspergillus versicolor based on the molecular characterization of $18 \mathrm{~s}$ rRNA gene. A simple and environmental benign approach for the biosynthesis of AgNPs using extracellular filtrate of Aspergillus Versicolor ENT7 as reducing agent has been reported. The synthesized AgNPs were well dispersed with the average size of $15.5 \mathrm{~nm}$, spherical in shape, high crystalline in nature, and highly stable with zeta potential of $-38.2 \mathrm{mV}$. The biosynthesized AgNPs exhibited a very good antioxidant and antimicrobial activity and clearly indicated their pharmaceutical and biomedical importance.

Acknowledgments We are thankful to Indian institute of chemical technology (IICT), Hyderabad, A.P., India for technical support.

\section{Compliance with ethical standards}

Conflict of interest The authors declared that they have no competing interest exists.

Open Access This article is distributed under the terms of the Creative Commons Attribution 4.0 International License (http:// creativecommons.org/licenses/by/4.0/), which permits unrestricted use, distribution, and reproduction in any medium, provided you give appropriate credit to the original author(s) and the source, provide a link to the Creative Commons license, and indicate if changes were made.

\section{References}

Asad S, Supriya S, Gopal CK, Absar A (2013) Biological synthesis of silver nanoparticles using the fungus Humicola sp. and evaluation of their cytotoxicity using normal and cancer cell lines. Spectrochim Acta Part A: Mol Bio Spec 114:144-147

Bozanic DK, Trandafilovic LV, Luyt AS, Djokovic V (2010) Green synthesis and optical properties of silver-chitosan complexes and nanocomposites. React Function Polym 70:869-873

Chen J, Luo Y, Liang Y, Jiang J, Shen G, Yu R (2009) Surfaceenhanced Raman scattering for immunoassay based on the biocatalytic production of silver nanoparticles. Anal Sci 25:347-352 
Chol SH, Zhang YP, Gopalan A, Lee KP, Khang HD (2005) Preparations of catalytically efficient precious metallic colloids by gamma irradiations and characterizations. Colloids and surf A: Physicochem Engg Asp 256:165-170

Cobley CM, Skrabalak SE, Campbel DJ, Xia Y (2009) Shape controlled synthesis of silver nanoparticles for plasmonic and sensing applications. Plasmonics 4:171-179

Edison T, Sethuraman M (2012) Instant green synthesis of silver nanoparticles using Terminalia chebula fruit extract and evaluation of their catalytic activity on reduction of Methylene Blue. Process Biochem 47:1351-1357

Fayaz AM, Balaji K, Kalaichelvan PT, Venkatesan R (2009) Fungal based synthesis of silver nanoparticles-an effect of temperature on the size of particles. Coll Surf B 74:123-126

Feng Q, Wu J, Chen G, Cui F, Kim T, Kim J (2000) A mechanistic study of antibacterial effect of silver ions on E. coli and $S$. aureus. J Bio Mate Res 52:662-668

Frattine A, Pellegri N, Nicastro D, De-Sanctis O (2005) Preparation of amine coated silver nanoparticles using triethylenetetraamine. Mater Chem Phys 94:148-152

Jaidev LR, Narasimha G (2010) Fungal mediated biosynthesis of silver nanoparticles, characterization and antimicrobial activity. Colloids Surf B 81:430-433

Javed M, Sourabh D, Singh BR, Abdulaziz AA, Ameer A, Alim N (2010) Production of antimicrobial silver nanoparticles in water extracts of the fungus Amylomyces rouxii strain KSU-09 Biores. Technol 101:8772-8776

Karimzadeh R, Mansour N (2010) The effect of concentration on the thermo-optical properties of colloidal silver nanoparticles. Opt Laser Technol 42:783-789

Kelly KL, Coronando E, Zhao LL, Schatz GC (2002) The Optical properties of metal nanoparticles: the influence of size, shape and dielectric environment. J Phys Chem B 107:668-677

Kim KJ, Sung WS, Suh BK, Moon SK, Choi JS, Kim JG, Lee DG (2009) Antifungal activity and mode of action of silver nanoparticles on Candida albicans. Biometals 22:235-242

Kotakadi VS, Rao YS, Gaddam SA, Prasad TNVKV, Reddy AV, Gopal DVRS (2013) Simple and rapid biosynthesis of stable silver nanoparticles using dried leaves of Catharanthus roseus Linn. G. Donn and its antimicrobial activity. Colloids Surf B Biointerfaces 105:194-198

Kotakadi VS, Aparna GS, Rao YS, Prasad TNVKV, Reddy AV, Gopal DVRS (2014) Biofabrication of silver nanoparticles using Andrographis paniculata. Eur J Med Chem 73:135-140

Kreibig U, Vollmer M (1995) Optical properties of metal clusters. Springer, Berlin, pp 483-525

Litvin VA, Minaev BF (2013) Spectroscopy study of silver nanoparticles fabrication using synthetic humic substances and their antimicrobial activity. Spectrochim Acta Part A Mol Biomol Spectrosc 108:115-122

Litvin VA, Galagan RL, Minaev BF (2012) Kinetic and mechanism formation of silver nanoparticles coated by synthetic humic substances. Colloids and surf A: Physicochem Engg Asp 414:234-243

Lok C, Ho C, Chen R, He Q, Yu W, Sun H, Tam PK, Chiu T, Che C (2006) Proteomic analysis of the mode of antibacterial action of silver nanoparticles. J Proteome Res 5:916-924
Longoria EC, Nestor ARV, Borja MA (2011) Biosynthesis of silver, gold and bimetallic nanoparticles using the filamentous fungus Neurospora crassa. Coll Surf B 83:42-48

Metuku RP, Pabba S, Burra S, Himabindu N, Gudikandula K, Singara charya MA (2014) Biosynthesis of silver nanoparticles from Schizophyllum radiatum HE 863742.1: their characterization and antimicrobial activity. 3 Biotech 4:227-234

Mittal AK, Kaler A, Banerjee UC (2006) Free radical scavenging and antioxidant activity of silver nanoparticles synthesized from flower extract of Rhododendron dauricum. Nano BioMed Eng 4:118-124

Monali G, Jayendra K, Avinash I, Aniket G, Mahendra R (2009) Fungus-mediated synthesis of silver nanoparticles and their activity against pathogenic fungi in combination with fluconoazole. Nanomed Nanotech Biol Med 5:382-386

Morones JR, Elechiguerra JL, Camacho A, Holt K, Kouri JB, Ramirez JT, Yacama JM (2005) The bactericidal effect of silver nanoparticles. Nanotechnology 16:2346-2353

Netala VR, KotakadiVS NagamV, Bobbu P, Ghosh SB, Tartte V (2014) First report of biomimetic synthesis of silver nanoparticles using aqueous callus extract of Centella asiatica and their antimicrobial activity. Appl Nanosci. doi:10.1007/s13204-0140374-6

Netala VR, Kotakadi VS, Domdi L, Gaddam SA, Venkata SK, Bobbu PL, Ghosh SB, Tartte V (2015a) Biogenic silver nanoparticles: efficient and effective antifungal agents. Appl Nanosci. doi:10. 1007/s13204-015-0463-1

Netala VR, Bobbu PL, Ghosh SB, Tartte V (2015b) Endophytic fungal assisted synthesis of silver nanoparticles, characterization, and antimicrobial activity. Asian J Pharm Clin Res 8:113-116

Plante IJL, Zeid TW, Yang P, Mokari T (2010) Synthesis of metal sulfide nanomaterials via thermal decomposition of single-source precursors. J Mater Chem 20:6612-6617

Pulicherla Y, Reddla H, Nataru S (2015) Synthesis, characterization and antimicrobial properties of green-synthesised silver nanoparticles from stem bark extract of Syzygium alternifolium (Wt.) Walp. 3 Biotech. doi:10.1007/s13205-015-0307-4

Rafie MH, Shaheen T, Mohamed AA, Hebeish A (2012) Biosynthesis and applications of silver nanoparticles onto cotton fabrics. Carbohydrate Pol 90:915-920

Ramamurthy CH, Padma M, Samadanam ID et al (2013) The extra cellular synthesis of gold and silver nanoparticles and their free radical scavenging and antibacterial properties. Colloids Surf B Biointerfaces 102:808-815

Rashmi S, Varma P (2009) Biomimetic synthesis and characterization of protein capped silver nanoparticles. Biores Technol 100:501-504

Ravindran A, Chandran P, Khan S (2013) Biofunctionalized silver nanoparticles: advances and prospects. Colloids Surf B 105:342-352

Simakin AV, Voronov VV, Kirichenko NA, Shafeev GA (2004) Nanoparticles produced by laser ablation of solids in liquid environment. Appl Phys A 79:1127

Strobel GA, Ford EJ, Worapong JK, Harper AM, Arif DM, Grant PC, Fung W, Chan K (2002) Ispoestacin, an isobenzofuranone from Pestalotiopsis microspora, possessing antifungal and antioxidant activities. Phytochemistry 60:179-183 